\title{
FUNDAMENTOS DE LA ACCIÓN PEDAGÓGICA EN EL ÁREA DE TECNOLOGÍA E INFORMÁTICA
}

\author{
Carlos Julio Romero \\ Profesor asociado Universidad Pedagógica \\ Nacional. \\ Evelio Nicanor Ortiz \\ Profesor Asistente Universidad Pedagógica \\ Nacional.
}

\begin{abstract}
Technology and Computer Science was included as a compulsory subject in the educational process in 1994. This inclusion has generated concerns in the institutions devoted to basic and intermediate education, giving rise to pedagogical, methodological and evaluation problems in the development of this field of knowledge.

The article draws attention to these aspects and begins considering the technology as a system related to elements such as the understanding of technology, the design, the production processes and the socio-environmental context, which are included taking into account 5 axes that promote an integrated and horizontal development of this field of knowledge, changing the way it is taught in the classroom. This is achieved by using a work methodology that comprises of problem solving and projects on specific themes. The article provides specificactions to be taken in the elementary school, basic high school and intermediate high school. It states the specific purpose of developing the capacity and the relevant skills that would enable progress. The goal is to go from an abstract concept to a concrete one, which properly represents the characteristic process that a person follows when dealing with technology. This pointed out as a way to focus the institutional work with the specific goal of progressing towards the emphases that have been declared as the target of the learning process.
\end{abstract}

\section{RESUMEN}

EL área de Tecnología e informática incorporada a partir de 1994 en el proceso educativo como obligatoria, ha generado en las instituciones de educación básica y media inquietudes frente a este campo del conocimiento, que ponen en evidencia dificultades de orden pedagógico, metodológico y evaluativo para el desarrollo del área.

El artículo centra su atención en estos aspectos y parte de la consideración de la tecnología como sistema, relacionando elementos tales como: el conocimiento, el diseño, los procesos de producción y el contexto socio-ambiental, los cuales, se involucran desde cinco ejes, que a partir de una metodología de trabajo por problemas y proyectos en torno a núcleos, posibilitan el desarrollo horizontal e integrado del conocimiento, cambiando la forma de trabajo en el aula.

Se establece acciones puntuales en la básica primaria, básica secundaria y la media para desarrollar habilidades y capacidades relevantes en el paso de lo abstracto a lo concreto como proceso característico de la tecnología. Lo anterior se plantea como una forma de centrar el trabajo institucional, con el propósito de avanzar hacia los énfasis constituidos en la meta del proceso de aprendizaje. 


\section{LA TECNOLOGÍA COMO SISTEMA}

La sociedad en general convive con desarrollos producto de la tecnología, beneficiándose directa 0 indirectamente de los que satisfacen sus necesidades particulares. Los derivados de dichos desarrollos (instrumentos) se utilizan dependiendo de los intereses, y son el medio que posibilita algún grado de satisfacción a determinada necesidad.

En el uso de un instrumento ${ }^{1}$ existe un fin expreso o tácito; para alcanzarlo se requiere establecer relación entre el usuario y el instrumento; relación que está dada por el nivel de interés del usuario, estableciéndose básicamente tres tipos de relación:

- Relación utilitaria; hace referencia a la posibilidad de uso del instrumento para una función específica.

- Relación operativa; hace alusión al dominio de los procesos técnicos propios para su producción, como también a los procedimientos de operación. El operario puede dar explicación sobre los procesos y procedimientos, de igual forma, hacer un reconocimiento del funcionamiento del instrumento.

Relación de conocimientos; hace referencia a la capacidad de apropiación y generación de saberes, exige investigación y argumentación. Se caracteriza porque hay un nivel explicativo desde los diferentes campos del conocimiento.

Aunque la relación con el instrumento tiende a lo específico, la tecnología no es enfática en un aspecto particular, involucra el conocimiento, el diseño, los procesos de producción, y el contexto socioambiental. Cada aspecto mencionado se entiende como:

A. Conocimiento: es esencialmente interdisciplinar y se entiende como el cuerpo de saberes que da razón del diseño, la construcción y el comportamiento de instrumentos y procesos tecnológicos, dentro de un ámbito de problemas, teorías, métodos, procedimientos y técnicas que le son propios.

El conocimiento tecnológico se dinamiza en la interacción que posibilita la reflexión en torno a una necesidad específica objeto de estudio. Por lo tanto, para desarrollar tecnología no es suficiente el manejo de conocimientos particulares y desarticulados; fundamentalmente es indispensable articular conocimientos desarrollados por diferentes campos.

B. Diseño: no es algo inmediato, es un proceso que depende de la capacidad del ser humano para generar estructuras concretas en torno a necesidades identificadas. Estructuras planteadas en primera instancia como un modelo teórico que establece características y condiciones para materializarlo. En la consolidación de dicho modelo el diseñador hace uso de conocimientos tecnológicos y no tecnológicos.

No se puede pensar en el modelo teórico que de pautas para que algo pueda ser, si no existe reflexión en torno al problema identificado, de igual forma, la reflexión para que el diseño se materialice no tiene sentido, si no está relacionado con el proceso de producción.

\footnotetext{
1 Instrumento: Todo aquello que posibilita alcanzar un propósito, para el caso particular, se refiere únicamente a los instrumentos producto de los desarrollos tecnológicos.
} 
El proceso de diseño se ejecuta en la consolidación del modelo teórico, el cual se ocupa, entre otros, del análisis formal, funcional y estructural. El primero hace referencia a los elementos externos (tangibles a los sentidos); el segundo, a la función y relación entre las partes, el último al comportamiento (físico, químico, mecánico, etc.) de cada elemento y del conjunto en su situación de trabajo. Lo anterior se concreta en primera instancia, a través de la expresión gráfica, proporcionando información sobre materiales, formas y dimensiones que junto con los procesos de producción permite la concreción en el prototipo.

En síntesis, el diseño es un proceso (Cuadro 1) intencional eminentemente cognitivo que se estructura en la consolidación del modelo teórico. Desde esta posición el diseño ${ }^{2}$ es la designación al método ${ }^{3}$ utilizado para hacer tecnología.

Cuadro 1. Proceso intencional eminentemente cognitivo.

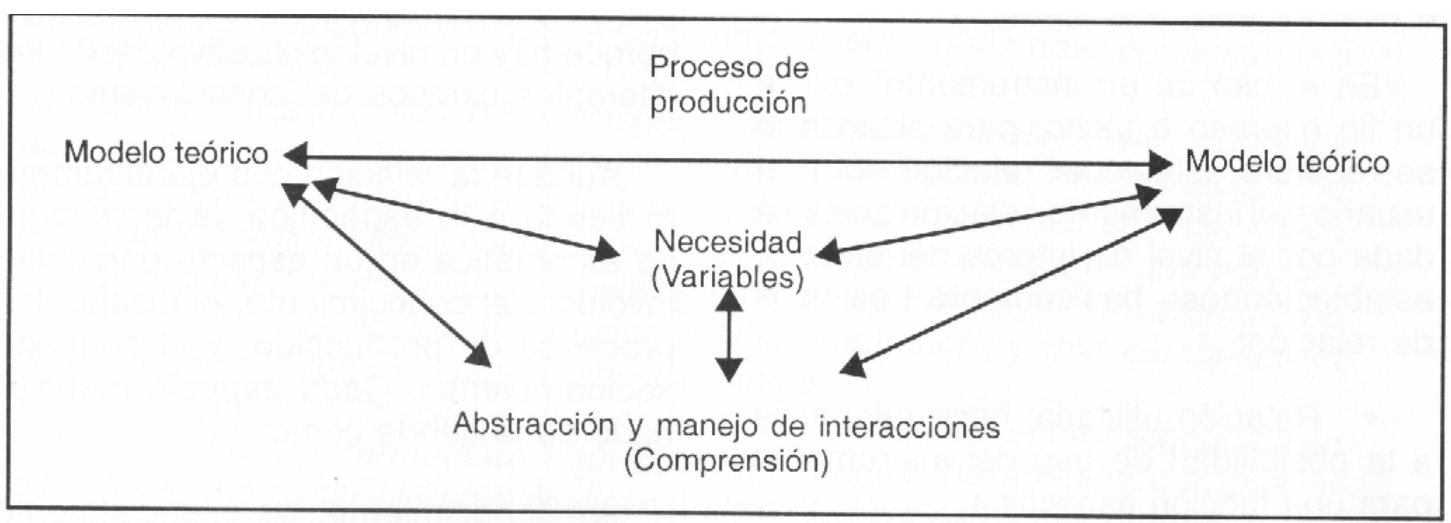

C. Procesos de producción: el estudio y comprensión de la necesidad puntual con el propósito de generar soluciones, determina las características de los materiales, los equipos, los procesos técnicos a desarrollar y las características del personal, las cuales, están relacionadas con los niveles de formación para la ejecución en el proceso.

D. Contexto socioambiental: En este espacio, se presentan tres componentes fundamentales; el social conformado por la interacción entre los individuos, el ambiental conformado por todos los sistemas naturales y el tecnológico constituido por los desarrollos científicos, tecnológicos y los instrumentos producto de esos desarrollos. La interacción entre el primero y el último componente ha alterado el sistema natural en detrimento de las posibilidades de supervivencia de la vida en todas sus manifestaciones; por lo cual, la formación en torno a los desarrollos tecnológicos no puede desconocer los impactos sociales y ambientales desde su producción hasta la eficiente utilización del producto y los deshechos.

De lo expuesto se podría concluir que la tecnología es un sistema, estructurado por el conocimiento, el diseño, los procesos de producción y el contexto socio-ambiental. Al interior del sistema se generan relaciones coherentes que posibilitan, explican y justifican la creación de procesos e instrumentos.

\footnotetext{
${ }^{2}$ El diseño no es un aspecto puntual que se pueda apreciare generar en un acto o en un instrumento, el diseño se constituye en el proceso.

${ }^{3}$ Entendido como la forma de análisis que genera interdisciplinariedad.
} 


\section{EJES DE TRABAJO PARA LA EDUCACIÓN BÁSICA Y MEDIA}

En el medio educativo, se ha centrado la atención en planteamientos teóricos sobre tecnología, aspecto importante, más no suficiente para el trabajo en la escuela. Esta, necesita de planteamientos que permitan implementar el área de tecnología en el salón de clase. El educador en tecnología, tiene como compromiso, desarrollar un proceso didáctico y metodológico que permita materializar los planteamientos teóricos en cuanto a educación en tecnología se refiere, para formar personas capaces de comprender ${ }^{4}$, pensar $^{5}$ y hacer tecnología.

Como base sobre la cual estructurar el plan de estudios en el área de tecnología e informática; se plantean ejes involucrados explícita o implícitamente en el sistema tecnológico, los cuales, son parte fundamental de la existencia y desarrolle de instrumentos que satisfacen necesidades de diferente índole. Los ejes ${ }^{6}$ propuestos son: energía, diseño, materiales, control y movimiento, y comunicación y maneje de información; los cuales se cementan a continuación:

- Materiales y energía: desde sus inicies la civilización ha centrado su actividad en los materiales y la energía. Los materiales han marcado grandes épocas en la evolución de la civilización. La utilización de les nuevos materiales ha transcurrido de la piedra y la madera al bronce, al hierro, al acero, a los sintéticos, a los organismos vives (para la manipulación genética) y en general a los diseñados con características especificas para cada necesidad. Por otro lado, el hombre ha transformado la explotación de las fuentes de energía, pasando de la obtenida por la actividad muscular directa, a la proporcionada por el agua y el viento en los molinos, posteriormente a la de las hidroeléctricas, a la solar, hasta la emancipación de la energía termonuclear.

- Comunicación y manejo de información: otro aspecto importante en la evolución de la civilización es la comunicación y difusión del conocimiento; que a partir de señales, el lenguaje oral y posteriormente el escrito, avanza de los manuscritos a la imprenta, y por último a la utilización de sistemas electrónicos. La comunicación y el manejo de información, en la actualidad, se constituyen en el núcleo del control social y en la base de los desarrollos científicos.

- Control y movimiento: Considerando que el desarrollo tecnológico partió de la satisfacción a diferentes necesidades, no se puede desconocer en este proceso la importancia del control y el movimiento. El movimiento como un factor inherente al funcionamiento de los instrumentos en contextos espacio-temporales definidos y el control, como el factor que permite determinar de forma específica la acción del instrumento en ese contexto.

- Diseño: Aspecto involucrado en toda solución, su estructuración y materialización solo es posible a través del proceso integrador del diseño.

Los anteriores son aspectos inherentes a los avances tecnológicos, que en sus diferentes manifestaciones interactúan con el hombre. La institución educativa a través de sus proyectos institucionales puede asumir con mayor dedicación aquellos aspectos en los que tiene fortalezas, o le corresponden particularmente como respuesta a su contexto.

\footnotetext{
${ }^{4}$ Me llama la atención el esplendor del universo, me fascina la perspectiva de comprender como funcionan realmente las cesas. Tomado de: Sagan, Carl. El mundo y sus demonios - La ciencia como una luz en la oscuridad. Santa Fe de Bogotá: Editorial Planeta. 1998, p. 43.

5 La ciencia es más que un cuerpo de conocimiento, es una manera de pensar". Ibid., p. 43.

${ }^{6}$ Ver Romero O., Carlos Julio y Ortiz CH. Evelio Nicanor. Area de tecnología e informática, p. 20.
} 


\section{RELACIÓN EJES Y ÉNFASIS}

Un aspecto claro, independientemente de los ejes que se designe como base para estructurar el trabajo en tecnología, es la necesidad de un alto nivel de integración y comprensión. Razones que permiten estructurar el plan de estudios para el área de tecnología e informática en torno a los énfasis y los ejes, en los cuales, adquieren gran importancia las habilidades, las capacidades y las competencias, consideradas como necesarias para acceder a mayores niveles de integración y comprensión. La formación está orientada por los énfasis ${ }^{7}$, que en lo general responden básicamente a la pregunta ¿qué personas quiere la institución al terminar el ciclo de formación secundaria? y en lo específico, ¿qué se pretende que los estudiantes hayan desarrollado al terminar cada nivel?; respuesta dada en los indicadores de logro.

En tecnología, un equipo de trabajo requiere de diferentes capacidades y competencias, para establecer una dinámica de pensamiento entre lo abstracto y lo concreto, por lo que es necesario, equipar al alumno con un mínimo de habilidades que permitan el desarrollo de capacidades y competencias; acción que empieza en los primeros años en la escuela. Las habilidades pueden conformar otras de mayor categoría denominadas capacidades, de tal manera, que la interacción de un conjunto de habilidades configuran una o más capacidades; las cuales, manejadas en términos creativos, de autonomía y adaptación a diferentes circunstancias, constituyen las competencias $^{8}$. Así, las capacidades y competencias son desarrollos necesarios para el análisis de situaciones, y la base fundamental de comprensión y caracterización de problemas factibles de solución tecnológica.

- En la investigación.

- En el manejo y proyección de la información.

Énfasis $\Rightarrow$ - En procesos interdisciplinarios.

- En el trabajo en equipo y la autonomía.

- En la construcción personal.

- En valores.

Es pertinente pensar en las capacidades y habilidades en el ámbito intelectual y motriz que puedan facilitar la estructuración del sistema tecnología, en el paso (caracterizado por el diseño) de lo abstracto a lo concreto. Así, la tecnología exige el desarrollo de un pensamiento integrador, fundamentado en la comprensión de principios, modelos teóricos y la estructuración y concreción de proyectos. Para que la praxis pueda ser, se plantean tres aspectos: conceptos, habilidades y técnicas, como unidad, desde la cual, la acción realizada con el alumno no es válida si falta un elemento de esta.

De tal manera, que los conceptos relacionados con las habilidades intelectuales en la integración y comprensión, potencian lo teórico, y las habilidades motrices con la técnica específica en la operación, potencia lo práctico. La interacción entre estos aspectos Conforma la unidad de acción en el Área de Tecnología.

\footnotetext{
${ }^{7}$ Ibid., p. 12.

NOTA: Los énfasis son importantes por el sentido que dan al área y las relaciones que involucran

${ }^{8}$ Hace referencia al conjunto de aspectos requeridos para que el acto sea eficiente e integral.
} 


\section{Unidad}

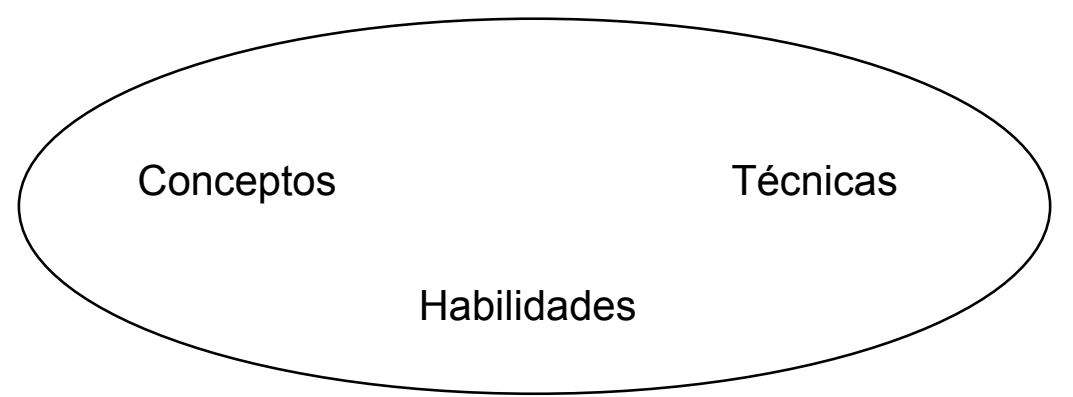

En la unidad se menciona únicamente habilidades por considerarlas como la base en el desarrollo de capacidades y competencias

En la generación de modelos teóricos con factibilidad de ser materializados es relevante la integración de conceptos, habilidades y técnicas, la comprensión de conceptos y técnicas y pensamientos estructurados que posean la dinámica para moverse entre lo abstracto y lo concreto. La reflexión que conlleva al desarrollo de tecnología inicia en la asimilación de una idea o necesidad (lo abstracto) y a través del proceso de diseño y producción culmina en lo concreto.

El paso de lo abstracto a lo concreto es un proceso característico de la tecnología, y requiere del desarrollo de capacidades y habilidades que posibilitan la estructuración de esa forma de pensamiento y la generación de procesos creativos.

Algunas capacidades significativas en este proceso son:

- Capacidad para la construcción de conocimiento y conservación del medio ambiente.

- Capacidad para experimentar.

- Capacidad para el manejo de información.

- Capacidad para trabajar en equipo.

- Capacidad para identificar situaciones y solucionar problemas.

- Capacidad para articular conocimientos en torno a un núcleo.

- Capacidad creativa.

- Capacidad para la cualificación y proyección personal.

- Capacidad para el reconocimiento y valoración del otro.

Las capacidades mencionadas no son las únicas; el hombre en el proceso de interacción involucra diferentes capacidades que supera suficientemente las referidas, lo cual, no indica mayor o menor importancia, esa categorización depende de la necesidad del individuo.

Entre las habilidades que apuntan al desarrollo de las capacidades mencionadas se plantean las siguientes: 


\section{HABILIDADES}

$\begin{array}{lllll}\text { Observa } & \text { Comprende } & \text { Plantea } & \text { Busca } & \text { Opera } \\ \text { Compara } & \text { Explica } & \text { Interpreta } & \text { Organiza } & \text { Manipula } \\ \text { Diferencia } & \text { Expresa } & \text { Elabora } & \text { Selecciona } & \text { Utiliza } \\ \text { Reconoce } & \text { Describe } & \text { Representa } & \text { Clasifica } & \text { Construye } \\ \text { identifica } & \text { Analiza } & & & \\ \text { Relaciona } & \text { Sintetiza } & & & \\ & \text { Argumenta } & & & \\ & & & \end{array}$

En el desarrollo de capacidades y habilidades se potencia el aprender a comprender, a cultivare desarrollar la creatividad y el use activo del conocimiento en el análisis de situaciones, la solución de problemas y el trabajo por proyectos, aspectos necesarios en el proceso característico de la tecnología.

\section{ORGANIZACIÓN DE LA ACCIÓN PEDAGÓGICA}

Se trata de la coordinación de las acciones del profesor y la orientación de las actividades a las que se va a enfrentar el alumno para alcanzar los prepósitos planteados.

Los problemas y proyectos ${ }^{9}$, son elementos que forman parte de la cotidianidad social, por lo cual, se constituyen en uno de los medios de mayor relación con la vida del alumno, para permitirle analizar, comprender y tomar decisiones en dicho contexto, el cual, no está organizado en los enfoques que las instituciones generan en torne al conocimiento y el aprendizaje, como lo muestra las acciones que ejerce la escuela, por ejemplo, en los tipos de bachillerato académico o clásico, técnicos y diversificados. Los de tipo clásico o académico se han centrado en un alto porcentaje en los conceptos, los de tipo técnico en las habilidades motrices y el manejo de técnicas, y los diversificados mantienen la misma tendencia con la diferencia que se centran en áreas especificas del conocimiento. En la actualidad, se habla de la importancia del desarrollo de competencias en la escuela, solo se espera que esta tendencia no genere otra particularidad de bachillerato.

Para el trabajo por problemas y proyectos como estrategia metodológica es pertinente subdividir la acción en los ciclos de básica primaria, básica secundaria y media, con la respectiva particularidad que se le confiere a cada uno. La acción metodológica en los niveles presenta tres mementos.

Uno corresponde a los aspectos necesarios para el trabajo por problemas y proyectos; otro, al desarrollo de conceptos, habilidades y técnicas como unidad que se mantiene desde la básica primaria hasta la media y el último, al desarrollo temático desde un núcleo

\footnotetext{
${ }^{9}$ En la metodología general se plantea el trabajo por problemas y proyectos, esto lleva a cuestionarse, ¿cuándo se trabaja por problemas y cuándo por proyectos? La diferencia radica, en que al hablar de problemas se busca centrar la atención en el análisis de los elementos que le constituyen, identificación de variables y relaciones, y planteamiento de alternativas de solución.

El trabajo por proyectes tiene como fin partir del análisis, el planteamiento de soluciones del eles problemas que se presenten hasta llegar necesariamente a la construcción tangible acorde a los requerimientos iniciales desde los cuales se partió; en síntesis es el conjunto de acciones desarrolladas en terno a une o varios problemas, que posibilitan el proceso particular que satisface el fin propuesto. Ibid., p. 45.
} 
que facilite la relación entre conceptos, habilidades y técnicas por un lado y por otro, al desarrollo temático de forma horizontal y no vertical, esto quiere decir, que se avanzará en los indicadores de logro planteados para cada uno de los ejes (energía, materiales. diseño, movimiento y control y expresión y comunicación) desde el comienzo de las actividades del año escolar.

En el primero, se involucra los niveles de básica y media. Para la básica primaria se enfatiza en el desarrollo de habilidades, para la básica secundaria en las capacidades y específicamente en el análisis de situaciones ${ }^{10}$, y para la media en las competencias y el desarrollo de proyectos.

El análisis de situaciones se constituye en la base fundamental de comprensión y caracterización de problemas. Para el trabajo por proyectos es necesario haber aprendido a identificar y analizar problemas. El analizar problemas exige el desarrolle de ciertas habilidades en la identificación de variables. Cabe aclarar, que lo propuesto en cada nivel no descarta ningún aspecto involucrado en los momentos planteados, solo se pretende establecer la secuencialidad que permita el trabajo por proyectos y evidenciar el avance en pro de los énfasis.

El segundo, involucra la característica de las actividades en cada nivel en lo referido a la relación que se establece entre indicadores de legro, habilidades (intelectuales y motrices), conceptos y técnicas, este requiere que en cada actividad ${ }^{11}$ esté presente la unidad regulada por los indicadores de $\operatorname{logro}^{12}$ y el trabajo en pro del desarrollo de capacidades y competencias.

El tercero, corresponde a la característica integradora de la actividad, que facilite el trabaje de los indicadores de logro, de tal suerte que no esté forzado ningún aspecto; esto lleva a no tener indicadores de logro puntuales en la distribución de los periodos del año escolar, sencillamente se tienen indicadores para el año escolar y se trabajan dependiendo de la actividad.

Los logros e indicadores de $\operatorname{logros}^{13}$, propuestos para cada eje, son únicamente aspectos necesarios para el trabajo en tecnología; no indican un orden; puesto que, el desarrollo puntual se gesta a partir del núcleo integrador. El núcleo puede ser una situación específica, un problema, un instrumento, un sistema, un documento, un plano, etc., lo importante es su orientación en relación con el propósito de cada nivel. Esquemáticamente se ilustra a continuación lo expuesto para la acción pedagógica.

\footnotetext{
${ }^{10}$ Con el análisis de situaciones se pretende, que el estudiante comprenda que la necesidad está inmersa en un contexto y se manifiesta por la carencia que siente el sujeto al interactuar en dicho contexto. Si el sujeto puede aislar e identificar con precisión en el contexto que se manifiesta la necesidad, las variables que lo estructuran y emprende acciones organizadas en la búsqueda de soluciones óptimas, ha identificado con claridad un problema. En estos términos se considera que la necesidad no constituye el problema, lo constituye la interacción entre las variables. Es precisamente, el control de las variables y sus interacciones lo que permite hablar de problemas y soluciones. De allí la importancia de enseñarle al alumno a analizar situaciones y problemas.

${ }^{11}$ La actividad estará planteada de tal manera que es un medio que contribuye al desarrollo de capacidades y competencias, enmarcada en los requerimientos de los énfasis.

${ }_{12}$ Los indicadores de logro tienen la característica de manejar habilidades, conceptos y técnicas a partir de la actividad planteada.

${ }^{13}$ Ver Romero C., Carlos Julio y Ortiz CH., Evelio Nicanor. Área de tecnología e informática... págs. 20-30. 


\section{METODOLOGIA}

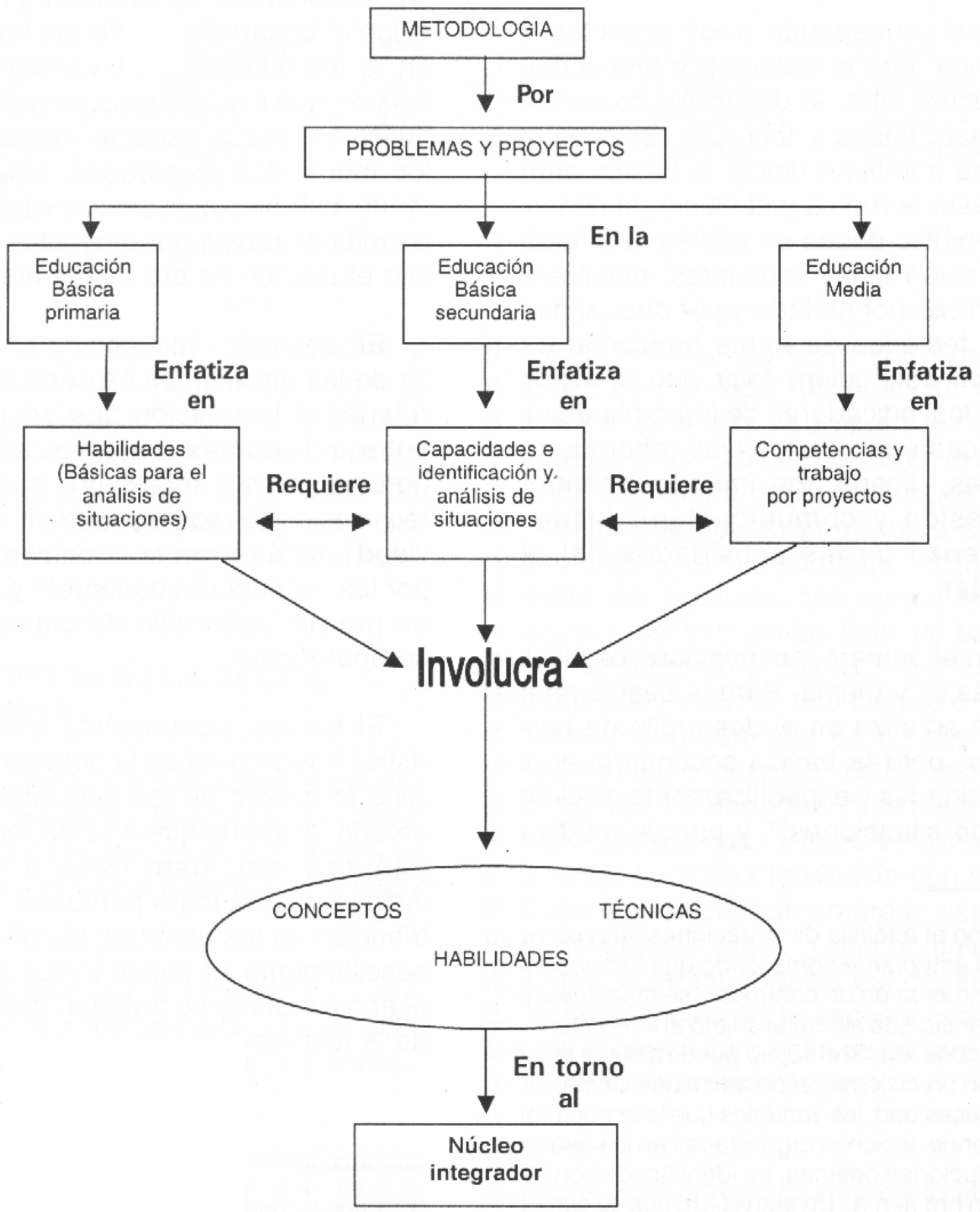

Análisis de situaciones en la básica secundaria ${ }^{14}$

${ }^{14}$ El análisis de situaciones en la básica secundaria involucra:

- El análisis.

- La caracterización de problemas.

- El proceso de solución. 
Como complemento a la orientación metodológica se precisa de las siguientes condiciones:

- No es válido el trabajo en el aula si se desarrolla únicamente habilidades, conceptos, o técnicas; la metodología debe permitir el trabajo integrado de estos aspectos.

- Desarrollar capacidades que influyen en el proceso característico de la tecnología (el paso de lo abstracto a lo concreto).

- Determinar para cada actividad habilidades y/o capacidades a desarrollar.

En lo específico las habilidades y capacidades se trabajan teniendo en cuenta el desarrollo del pensamiento del alumno. No se trata de parcelar las habilidades en el desarrollo de una actividad, se trata de recalcar en las que tiene mayor recepción el alumno debido a su estado de desarrollo cognitivo. Por ejemplo, no sería muy productivo enfatizar en la síntesis con alumnos de la básica primaria, es de mayor provecho orientar el trabajo hacia el desarrollo de la observación.

Alcanzar lo propuesto en la metodología depende de la planificación de las actividades. Para apoyar la habilidad que se busca desarrollar, no es suficiente con decirle al alumno observe o describa, es indispensable puntualizar a través de la actividad, en una observación o descripción organizada que le permita ir apropiando el proceso y los elementos básicos para avanzar a lo más complejo de la observación, de igual forma con toda habilidad y capacidad que se pretenda desarrollar.

\section{SEGUIMIENTO DEL PROCESO DE APRENDIZAJE}

La evaluación como parte del proceso de seguimiento, de formación y construcción del ser humano, proporciona información que ha de ser útil en la toma de decisiones respecto a las nuevas acciones que permitirán alcanzar los logros propuestos. En este sentido es pertinente mirar mas que un resultado final, el proceso y los acontecimientos que llevan a dicho resultado, lo que implica establecer momentos de control e instrumentos funcionales y ágiles para el registro de información.

En el ámbito escolar, la evaluación se constituye en parte del proceso de aprendizaje, con propósitos claramente definidos en la ley general de educación, en el decreto 1860 de 1994 reglamentario de dicha ley y en la resolución 2343 de 1996.

En el área de tecnología e informática el proceso de evaluación debe estar en concordancia con los fines del área, las metodologías asumidas y el referente legal, de tal manera, que los instrumentos aporten información sobre el estado y evolución del estudiante, en cuanto a las habilidades tanto intelectuales como motrices propuestas en los logros; con el propósito de establecer nuevas acciones que apoyen el desarrollo de los estudiantes.

Para lo cual, es necesario tener presente, que la evaluación es llamada a cumplir con aspectos específicos de $\operatorname{ser}^{15}$ :

\footnotetext{
${ }^{15}$ MINISTERIO DE EDUCACIÓN NACIONAL. Serie documentos de trabajo. "La evaluación en el aula y más allá de ella". Lineamientos para la educación preescolar básica y media. GRUPO DE ESTÁNDARES Y EVALUACION DE LOGRO ACADÉMICO Y FACTORES ASOCIADOS. Santa Fe de Bogotá, D. O., abril de 1997, p. 25.
} 
- Continua: Que el seguimiento se constituya en un proceso en el espacio y en el tiempo.

- Integral: Que no descarte ninguna dimensión del ser humano; está indicando que lo flexible sobre todo está referido a lo integral.

- Sistemática: que exista relación entre indicadores de logro, metodología, objetivos del plan de estudios y fines pedagógicos, y los instrumentos de recolección de información.

- Flexible: que tiene en cuenta "ritmos de desarrollo, intereses, capacidades, limitaciones y... su situación concreta".

- Interpretativa: que interpreta (da significado) el proceso desarrollado por el alumno.

Al integrar los 5 aspectos para su aplicación, se parte de reconocer que la estructura de los instrumentos es el resultado de la acción sistemática en torno al problema pedagógico de educar y de evaluar, lo cual, garantiza la relación con los indicadores de logro, la metodología, los objetivos del plan de estudios y los fines pedagógicos. Los instrumentos que garantizan la exigencia de lo sistemático se aplican de forma continua espaciotemporalmente, e interiorizan la flexibilidad en cuanto no descartan ningún aspecto del ser humano factible de ser evaluado en el contexto escolar, es decir, son integrales.

La lectura de la información recopilada no puede ceñirse únicamente a la estadística, exige flexibilidad en su interpretación dependiendo de la particularidad del alumno.

En el documento Evaluación en el aula y más allá de ella, se plantea que, "la evaluación de los procesos de desarrollo del alumno se refiere a los aspectos biológicos, comunicativo, cognoscitivo, valorativo y afectivo" ${ }^{16}$, entendiendo que son aspectos que conducen a lo integral, incluyendo en:

- Lo biológico: desarrollo muscular, el movimiento, la coordinación, el equilibrio, la salud, la higiene, etcétera.

- Lo comunicativo: capacidad para manejar y comprender distintas formas de expresión, producir y captar mensajes, manejar códigos y sistemas simbólicos, construir y comprender significados.

- Lo cognoscitivo: desarrollo del pensamiento que implica actividades como la atención, la percepción, el análisis, la síntesis, la solución de problemas, la construcción y manejo de conceptos, principios y métodos, entre otros. El manejo de conceptos implica necesariamente el desarrollo de análisis, síntesis y los demás relacionados en el campo de habilidades.

- Lo valorativo: entre otros, los valores morales y estéticos. En los valores morales; la formación del sentido de justicia, la observancia de las normas, la participación, al respeto de los derechos humanos, la tolerancia, la convivencia, el desarrollo de la autonomía y, en general el desarrollo de la conciencia moral y ética. En los valores estéticos, el desarrollo de la sensibilidad, la expresividad, la emotividad, el gusto, etcétera.

- Lo afectivo: Los sentimientos, las emociones y las actitudes.

Estos por lo general están presentes de forma continua en las actividades del hombre, pero no es procedente evaluar en cada actividad todo lo que pueda involucrarse en la misma, puesto que no es factible para un docente con un alto número de estudiantes. El profesor estaría imposibilitado para realizar la evaluación dentro de los parámetros exigidos, porque además de orientar y explicar lo específico tiene que evaluar todos los elementos involucrados en lo integral.

${ }^{16}$ Ibíd., pp. 24-25. 
La diversidad de aspectos que involucra lo integral obliga a centrar la atención en factores particulares pertinentes a cada área, lo que no implica que las demás áreas y profesores se desentiendan, solo indica que no serán objeto específico de los instrumentos de recolección de información, por lo tanto, no incidirá de forma definitiva en la promoción, por el contrario, se constituirán en casos de orientación constante a través del diálogo y reporte a las áreas de mayor afinidad, por ejemplo, si el trabajo está en torno a los derechos humanos, y se ha identificado un problema de motricidad fina en uno o varios alumnos, aunque se de la orientación específica no se profundizará al respecto y no podría prevalecer este hecho como factor decisivo en la aprobación o reprobación. El profesor no es ni podrá ser especialista en todos los campos. De igual manera, existen aspectos que pueden ser responsabilidad de todos los profesores y de todas las áreas, los cuales deben acordarse.

Lo anterior se evidencia si se establece la relación profesor número de alumnos y aspectos a evaluar. Como lo integral involucra diversidad de aspectos, en la práctica es imposible realizar una evaluación integral y alcanzar los propósitos de la misma sin cambiar la forma como está organizada en la actualidad, entonces: ¿cómo cumplir con las exigencias realizadas al proceso de evaluación?

En primer lugar, es necesario comprender que lo integral no puede verse desde la acción evaluativa de un profesor si no, desde la estructura de los planes de estudio, el currículo y la estructura de cada establecimiento educativo. Como la escuela no es un profesor, un alumno o un área, por el contrario, es un conjunto constituido por profesores, alumnos, currículo, etc., esto conduce a que cada área por afinidad con algún aspecto que involucra lo integral, lo evalúe desde allí, es decir, exprese el informe de aspectos afines que influyen de forma relevante en el proceso. Posición que plantea la necesidad de reconocer la escuela como un sistema, en el cual, la acción de cada componente adquiere importancia en la medida que reconozca la función de los elementos que lo integran y actúe interrelacionadamente en pro del conjunto.

En segundo lugar, concebir la evaluación inscrita en la acción del docente. En la práctica, el alumno presenta estados iniciales en el manejo de conceptos que puede o no comprender, es decir, puede variar el nivel de comprensión de algo que le permite desenvolverse en la vida, y que es factible que no corresponda a ningún logro e indicador planteado en los planes de estudio de la escuela. Para no buscar qué logros e indicadores pueden estar relacionados en la actividad del alumno, se busca actividades familiares a él, a través de las cuales, se desarrollan acciones para aproximarse o superar el referente explícito en los logros.

El avance entre los estados iniciales y el referente se manifiesta en las argumentaciones, las explicaciones, el análisis de problemas y el desarrollo de proyectos.

En tercer lugar, la inclusión de la evaluación en la estructura curricular, no como la descripción de características y de lo que debe ser, si no, como una estrategia que relaciona los propósitos de las áreas, los planteamientos metodológicos y los indicadores. En pro de esta idea se entiende que el proceso de evaluación en general está llamado a cumplir con unos requisitos para que adquiera sentido o razón de ser, entre estos se plantea $^{17}$.

\footnotetext{
${ }^{17}$ MINISTERIO DE EDUCACIÓN NACIONAL. Serie documentos de trabajo. "La evaluación en el aula y más allá de ella". Lineamientos para la educación preescolar básica y media. GRUPO DE ESTANDARES Y EVALUACIÓN DE LOGRO ACADÉMICO Y FACTORES ASOCIADOS. Santa Fe de Bogotá, D. O., abril de 1997, p. 22.
} 
Referidos al docente:

- Diagnosticar y pronosticar las tendencias del alumno.

- Identificar ritmos y estilos de aprendizaje.

- Identificar dificultades

- Proporcionar información para tomar decisiones.

- Mantener o reorientar y mejorar la calidad del proceso educativo.

- Promover o no de grado

Referidos al alumno:

- Permitirle aprender de la experiencia.

- Facilitarle identificar deficiencias y fortalecer aciertos.

- Reconocer el estado de su proceso.

Los anteriores requisitos, tanto los referidos al docente como al alumno están relacionados con la búsqueda de información para lo cual se utilizará algún tipo de estrategia o técnica puntual.

La información recopilada en el proceso de evaluación, se somete a organización y análisis para emitir las respectivas argumentaciones sin el carácter calificativo que ha asimilado la escuela, relacionado más con el valor del producto. El proceso de evaluación involucra elementos como los relacionados en el siguiente cuadro, en el que se muestra el planteamiento ideal y la práctica del docente en el aula.

Teniendo presente los aspectos involucrados en la evaluación (integral, continua, sistemática, flexible, interpretativa, participativa) para cumplir con los fines que avalan su razón de ser, es pertinente:

- Diferenciar los logros e indicadores de logro relacionados con la unidad (habilidades, conceptos y técnicas) de los de carácter eminentemente comportamental.

- Identificar los aspectos factibles de seguimiento y evaluación desde todas las áreas y profesores, y aquellos de seguimiento particular desde cada área.

- identificar los aspectos de carácter comportamental y/o actitudinal que van a influir en la decisión de promoción y permanencia en la institución.

Teniendo en cuenta las anteriores precisiones surge la pregunta ¿qué se va a evaluar? La respuesta no puede ser el logro e indicadores de logro, éstos pueden adquirir múltiples sentidos dependiendo de lo que cada actuante pueda ver en esos planteamientos, conduciendo al cuestionamiento, ¿en qué centra la acción el docente?; la respuesta de ésta conduce a la respuesta de la primera; en:

- La integración entre habilidades, conceptos y técnicas entorno a un núcleo, derivando el desarrollo de capacidades y competencias. La integración se entiende como las relaciones que se pueden establecer entre los aspectos mencionados, de tal forma, que no permite la concepción de teoría y práctica como dos aspectos independientes, sino del conjunto de acción frente a una situación que origina un problema.

- El trabajo en equipo.

- El análisis de situaciones, el trabajo por problemas y el trabajo en proyectos; este aspecto se enfoca a analizar y resolver problemas factibles de solución tecnológica 
Finalmente es necesario plantear la estrategia e instrumentos para consignar información y evaluar la acción del estudiante en los momentos que se determine para el control:

Es claro que no se puede desligar la estrategia de los instrumentos, esto sugiere que el instrumento tiene implícita la estrategia. La estrategia para realizar el seguimiento del proceso de aprendizaje del estudiante en el paso de lo abstracto a lo concreto (proceso característico de la tecnología) plantea:

a. Orientar al alumno para que aprenda a reflexionar sobre lo que hace, a analizar sus desarrollos y consignar la información producto del análisis y de sus actividades en el área de tecnología de forma organizada, de tal manera que le permita visualizar su proceso a él como al profesor, para lo cual el instrumento es la carpeta.

\section{EL PROCESO PRESENTA}

\begin{tabular}{|c|c|c|c|}
\hline ACCIONES & \multicolumn{2}{|c|}{ LO IDEAL } & LO OUE SE DA \\
\hline & ESTRATEGIAS & TÉCNICAS & \\
\hline $\begin{array}{l}\text { Búsqueda de } \\
\text { información }\end{array}$ & $\begin{array}{l}\text { Heteroevaluación } \\
\text { Autoevaluación } \\
\text { Coevaluación }\end{array}$ & 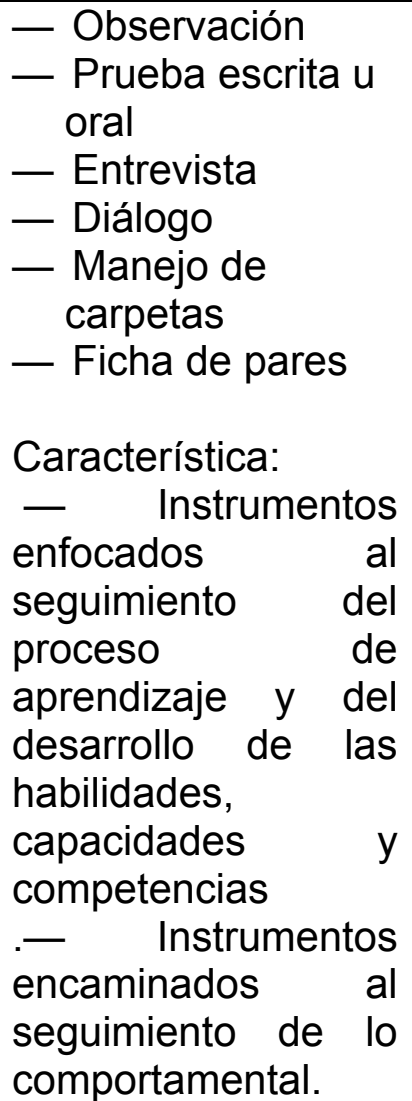 & $\begin{array}{l}\text { La heteroevaluación a } \\
\text { través de la forma } \\
\text { escrita, oral, de } \\
\text { exposiciones, } \\
\text { trabajos escritos, etc., } \\
\text { con mínima o ninguna } \\
\text { sistematización. }\end{array}$ \\
\hline $\begin{array}{l}\text { Organización y } \\
\text { análisis }\end{array}$ & $\begin{array}{l}\text { Establecimiento } \\
\text { criterios }\end{array}$ & $\begin{array}{l}\text { Las establecidas } \\
\text { para el manejo de } \\
\text { información. }\end{array}$ & $\begin{array}{l}\text { Generalmente no se } \\
\text { realiza. }\end{array}$ \\
\hline $\begin{array}{l}\text { Expresión } \\
\text { (manifestación) }\end{array}$ & $\begin{array}{l}\text { Con argumentos que } \\
\text { en algún momento } \\
\text { dado se convierten en }\end{array}$ & $\begin{array}{l}\text { Interpretación del } \\
\text { estado del proceso } \\
\text { de cada alumno, }\end{array}$ & $\begin{array}{l}\text { Los indicadores de logro } \\
\text { con- } \\
\text { notados con el rango }\end{array}$ \\
\hline
\end{tabular}


UNIVERSIDAD PEDAGÓGICA NACIONAL

\begin{tabular}{|c|c|c|c|}
\hline & juicios valorativos. & $\begin{array}{l}\text { expresados a los } \\
\text { padres y alumnos } \\
\text { en informes } \\
\text { descriptivos r y } \\
\text { comprensibles. }\end{array}$ & $\left.\begin{array}{llll}\text { estipulado } & \text { en } & \text { la } \\
\text { actualidad } & (\mathrm{E}, & \mathrm{B} . & \mathrm{I}\end{array}\right)^{18}$ \\
\hline $\begin{array}{ll}\text { Toma } & \text { de } \\
\text { decisiones } & \end{array}$ & $\begin{array}{l}\text { - Comparación entre } \\
\text { el estado del proceso } \\
\text { de aprendizaje del } \\
\text { alumno y los } \\
\text { indicadores de logro. } \\
\text { - Diferenciación de } \\
\text { los logros } \\
\text { indicadores de logro } \\
\text { involucrados en el } \\
\text { proceso } \\
\text { aprendizaje } \\
\text { especifico, de los de } \\
\text { carácter } \\
\text { comportamental. }\end{array}$ & $\begin{array}{l}\text { - Promoción o } \\
\text { ratificación en el } \\
\text { grado. } \\
\text { - Planteamiento de } \\
\text { actividades } \\
\text { complementarías, } \\
\text { - Consolidación o } \\
\text { cambio de la } \\
\text { práctica pedagógica. }\end{array}$ & $\begin{array}{l}\text { Aprobación } \\
\text { reprobación sin el } \\
\text { análisis de los informes } \\
\text { periódicos del proceso } \\
\text { de cada estudiante. Los } \\
\text { informes descriptivos y } \\
\text { explicativos no existen. }\end{array}$ \\
\hline
\end{tabular}

b. La observación sistemática por parte del profesor de aspectos ${ }^{19}$ debidamente determinados, además, como la metodología propone el trabajo en equipo, se requiere involucrar a los alumnos como observadores del proceso de sus compañeros. El instrumento para la observación debe ajustarse a las exigencias de cada situación específica, por lo tanto tiene que diseñarlo el profesor.

c. Brindarle la oportunidad al alumno de reflexionar sobre las conceptualizaciones que realiza y cómo esos conceptos le son útiles para desenvolverse y comprender el mundo como su entorno inmediato, para esto se propone la ficha de pares.

d. Que el profesor asimile el manejo de lo que se propone y oriente pausadamente a los alumnos al manejo de los instrumentos, de tal manera, que ellos conciban la importancia que tienen los instrumentos para el seguimiento de su proceso. Recordamos que esto se inicia igualmente como un proceso que requiere determinado tiempo.

Así, la observación y el registro por parte del docente y el estudiante, el diligenciamiento de la carpeta memoria de trabajo por parte del estudiante y el diligenciamiento de la ficha de pares, se plantean como instrumentos básicos para el seguimiento del proceso de evolución del aprendizaje en el área de tecnología e informática.

Cuando se trabaja a partir de problemas como estrategia metodológica para la apropiación de conocimientos, se establecen relaciones entre diferentes campos involucrados en la solución. El desarrollo del problema va aportando elementos que enriquecen el conocimiento de quien lo trabaja. El registro más efectivo de evidencias sobre la evolución del problema lo constituye el desarrollo de carpetas. Entendidas como el compendio de información relacionada con el problema objeto de trabajo, que organizadas por el estudiante desde el momento que inicia la actividad, suministran

\footnotetext{
${ }^{18}$ Algunas instituciones realizan esfuerzos por presentar informes descriptivos y explicativos del avance del alumno.

${ }^{19}$ Hace referencia entre otros a la observación de habilidades, capacidades, técnicas y actitudes.
} 
diferentes elementos sobre logros de los estudiantes, así como información sobre sus respuestas, avances y reflexiones.

Por otra parte, el mismo proceso lo estará observando el docente, para lo cual, necesariamente tiene que determinar las habilidades y/o capacidades, en concordancia con la actividad desarrollada, y realizar los registros correspondientes en una matriz que contenga los indicadores de logro definidos para la actividad. La observación se centrará específicamente en los aspectos que el docente pretende desarrollar.

\section{LA CARPETA}

Como instrumento para organizar la información y registrar la evolución del problema, puede tener diferente aspecto y contener múltiple información. Con relación a la forma, es un buen ejercicio dar libertad para definirla; en cuanto a la información, es pertinente precisar que está estrechamente relacionada con un método proyectual. Al respecto, se propone como elementos fundamentales los siguientes:

- DEFINICION: Plantea claramente la necesidad que origina el problema.

- PROBLEMA: Expresa claramente el problema a trabajar.

- PERTINENCIAS: Relaciona los aspectos o factores que le son propios al problema y determinantes para la solución.

- ACOPIO DE INFORMACIÓN: Desde todas las fuentes posibles presenta la información que permite reconocer el estado de desarrollo del problema trabajado (libros, revistas, catálogos, fotos, periódicos, etc.).

- PROPUESTA O ALTERNATIVA DE SOLUCIÓN: A través de la expresión gráfica a mano alzada se presenta las propuestas para su análisis funcional. Se complementa el modelo teórico con el análisis formal y estructural.

- EXPRESIÓN GRÁFICA (Planos de fabricación): Proporciona la información sobre formas, dimensiones y materiales (expresa el modelo teórico en lenguaje gráfico), para la construcción del prototipo.

- MÉTODOS DE APROXIMACIÓN: Permite el análisis formal y funcional previo al prototipo, sobre configuraciones a escala y con diferentes materiales; conocidos como maqueta si muestra solo condiciones volumétricas y modelo si representa en detalle cada elemento de la solución.

- PROTOTIPO: Configuración que responde al modelo teórico, elaborado a través de procesos de manufactura previamente determinados. Es la respuesta al problema planteado.

Los elementos propuestos se trabajan teniendo en cuenta el manejo y desarrollo de los estudiantes, así como el nivel de formación en el que se encuentran; de tal forma que puede ir desde simples descripciones hasta el análisis, desde simples bosquejos hasta planos técnicos, desde manualidades hasta prototipos.

Por lo anterior, el énfasis para básica primaria estará en:

- Reconocimiento de necesidades en diferentes situaciones.

- Acopio de información.

- Propuestas o alternativas de solución enfatizando en lo formal y lo funcional.

- Métodos de aproximación. 
Para la básica secundaria el énfasis estará en:

- La formulación y definición de problemas.

- Determinación de pertinencias.

- Acopio de información.

- Propuestas o alternativas de solución; con mayor exigencia en la expresión gráfica, en el análisis formal, funcional y estructural.

- Expresión gráfica.

- Métodos de aproximación.

En la educación media se trabajará todos los elementos propuestos, con énfasis en la confrontación entre el modelo teórico y el prototipo.

En el seguimiento y puesta en común de los avances de la carpeta ${ }^{20}$, se ha de tener en cuenta:

- Identificación de necesidades.

- Generación de alternativas.

- Planeación y fabricación.

- Respuesta tangible a la necesidad.

- Aprovechamiento de información.

\section{LA FICHA DE PARES}

El instrumento busca indagar sobre la comprensión de los conceptos. Utilizando pares de conceptos permite la relación, la diferenciación, la explicación, el establecimiento de analogías y la identificación de la aplicación de los conceptos en lo cotidiano del alumno.

A través del instrumento se pretende que el alumno tenga la posibilidad de pensar y volver a pensar sobre los conceptos trabajados y sus relaciones, de tener claridad sobre los aspectos débiles y fuertes, de desarrollar criterios de análisis, de retroalimentación acorde a sus dificultades y de apoyo al proceso de aprendizaje, si se entiende la actividad como un elemento constante que permite la reflexión.

La ficha de pares permite reforzar la interacción grupal, la autonomía, y la retroalimentación. Al profesor le facilita la coevaluación y la heteroevalaución.

La interacción grupal, porque permite a los integrantes exponer argumentos a partir de las respuestas, y en equipo, volver a plantear las respuestas de la ficha de pares.

La autonomía en la medida que adquiere criterios de crítica con argumentos del trabajo de los compañeros como de su propio desempeño. Esto le permite aprender a reconocer el trabajo del otro, a comprometerse académicamente con el otro y aprender a aceptar la crítica.

La retroalimentación, porque a partir de los argumentos de los compañeros pueden ampliar, modificar, aclarar y reflexionar sobre lo propio.

\footnotetext{
${ }^{20}$ La puesta en común o plenarias, permiten socializar el trabajo de cada carpeta y da la oportunidad de reflexionar y discutir sobre las propuestas y los avances en ellas consignados. Culminada la actividad, la carpeta se convierte en un referente para nuevos trabajos o cualificación de los ya desarrollados.
} 
La coevaluación, en cuanto se puede analizar el desarrollo de la ficha de pares en parejas, en grupos determinados por el alumno o el profesor o en el conjunto de la clase; o por el contrario, los argumentos y calificativos pueden ser manifestados únicamente por el profesor; en este caso se estaría practicando la heteroevalución, en tanto que es el profesor valorando el desempeño de varios alumnos.

En síntesis la actividad brinda al alumno la oportunidad de reflexión sobre su progreso e identificación de dificultades para la comprensión de los conceptos trabajados.

FICHA DE PARES (Instrumento)

Nombre del alumno

Curso

Temática 1.

Temática 2.

\begin{tabular}{|l|l|l|}
\hline & PLANTEAMIENTO & DIFICULTAD \\
\hline $\begin{array}{l}\text { No. } 1 \\
\text { Definición } \\
\text { No.2 }\end{array}$ & & \\
\hline Relación & & \\
\hline Diferenciación & & \\
\hline Aplicación & & \\
\hline $\begin{array}{l}\text { Conceptualización } \\
\text { de No. 1 y No. 2 }\end{array}$ & & \\
\hline
\end{tabular}

Los ítems del instrumento hacen referencia a:

Temática 1 y 2: Se presentan los aspectos puntuales a trabajar sobre los cuales se expresarán los conceptos.

Definición ${ }^{21}$ No. 1 y 2: El estudiante presenta las definiciones relacionados con las temáticas 1 y 2 , como las encuentra en las consultas y síntesis realizadas.

Relación: Expresa las relaciones que establece entre los dos conceptos a trabajar.

Diferenciación: Plantea las características que permiten manifestar que los dos conceptos trabajados son diferentes. Se pretende que el profesor no se quede en las diferencias y relaciones de forma y avance a las estructurales.

\footnotetext{
${ }^{21}$ Parte en la que el alumno escribe las definiciones recopiladas a partir de los textos o de las explicaciones del profesor. Al hacer referencia a conceptos, se busca indagar sobre lo que ha podido reconstruir, sobre los manejos y profundidad que ha alcanzado. Se presenta la aclaración porque consideramos, que si únicamente se repite el copiado a partir de las consultas o explicaciones es un manejo impersonal que no influye en la comprensión de lo aprendido, del mundo, nido su entorno inmediato, en tanto que el concepto, implica procesamiento de la información y comprensión por parte del alumno, esto indica que no repite de la misma forma, si no que genera procesos de reconstrucción de los conceptos acorde a las necesidades.
} 
Conceptualización: Manifiesta el concepto de las temáticas planteadas; amplía y sustenta tanto las relaciones como las diferencias

Utilización o aplicación: Expresa la aplicación de los conceptos en la acción diaria del alumno o cómo los ha utilizado o en qué circunstancias ha observado que se utilizan.

PLANTEAMIENTO: Corresponde a los espacios en los cuales el alumno manifestará lo que sabe de las temáticas planteadas.

DIFICULTAD: Los espacios correspondientes a esta columna están disponibles para que el alumno explique las dificultades que se le presentan en el manejo de los conceptos $y$ en el proceso se concientice de lo que se le dificulta manejar.

Un manejo reflexivo y detenido sobre las temáticas fortalece al alumno para elaborar y expresar con argumentos consistentes los conceptos que asimila. Para lo cual, es necesario que el alumno desarrolle muchas actividades que le permitan afianzar de forma creativa los con-coptos que trabaja.

El análisis de la información del proceso de aprendizaje debe involucrar la información recopilada por medio de la observación, la carpeta y la ficha de pares, con el fin de elaborar cuidadosas representaciones del progreso de cada alumno. La responsabilidad de esta actividad debe ser compartida entre docentes y estudiantes.

\section{BIBLIOGRAFÍA}

Acevedo Díaz, José Antonio. "Análisis de algunos criterios para diferenciar entre ciencia y tecnología". EN: Enseñanza de la ciencia. Vol. 16. No. 3. Nov. 1998.

Charum, Jorge. "El conocimiento tecnológico y la formación. Su relación con el trabajo". En: Educación trabajo y transformaciones tecnológicas en Colombia. Situaciones y perspectivas. Memorias seminario nacional realizado en Cali. 16 y 18 de mayo de 1990.

De Gortari, Elí. Indagación crítica de la ciencia y de la tecnología. México: Editorial Grijalbo. 1994.

De Zubiría Samper, Julián. Tratado de pedagogía conceptual. Los modelos pedagógicos. Colombia: Editorial Famidi, 1994.

Delors, Jacques. La educación encierra un tesoro. Informe a la UNESCO de la Comisión Internacional sobre la educación para el siglo XXI. España: Santillana Ediciones UNESCO. 1996.

Flórez Ochoa, Rafael. Hacia una pedagogía del conocimiento. Editorial Mc Graw Hill, mayo 1997.

Jara, N., María Inés. Introducción al conocimiento científico y filosófico. 1993.

Johnson, David W. y Johnson, Roger J. Aprender juntos y solos. Aprendizaje cooperativo, competitivo e individualista. AIQUE Grupo editor SA. Capital federal. Libro de edición Argentina. 1999. 
M.E.N. Ley general de educación. Ley 115 de 1994.

. Resolución Número 2343 de junio 5 de 1996.

Serie documentos de trabajo. La evaluación en el aula y más allá de ella. Lineamientos para la educación preescolar, básica y media. Santa Fe de Bogotá, D.C., abril de 1997.

M.E.N. Decreto 1860 de agosto 3 de 1994. Reglamentario de la ley 115 del 8 de febrero de 1994.

M.E.N., UNIVERSIDAD PEDAGÓGICA NACIONAL, et. al. Memorias Primer Congreso Colombiano y Primero Latinoamericano de Educación en Tecnología. Bogotá. 1997.

Munin, Helena (comp.). La autonomía de la escuela: ¿libertad y equidad? Un recorrido por la discusión alemana de los años noventa. Argentina: AIQUE Grupo editor SA. Capital federal. Libro de edición. 1999.

Pereda, Martín JA. Nuevas tecnologías y cambio social. Universidad Complutense de Madrid. Editorial El Escorial. 1989.

Pérez Calderón, José Unas y Romero Castro, Carlos Julio. Una estrategia pedagógica para el taller básico integral en el Departamento de Tecnología. Bogotá: Universidad Pedagógica Nacional. 1985.

Pérez Calderón, José U rías. Educación, tecnología y desarrollo. Bogotá: Panamericana, 1987.

Perkins, David. La escuela inteligente. Del adiestramiento a la educación de la mente. Barcelona: Editorial Gedisa. 1997.

Porlan, Rafael y Martin, José. El diario del profesor. Un recurso para la investigación en el aula. Edición 5. Sevilla: Diada Editora S.L., 1997.

UNIVERSIDAD PEDAGÓGICA NACIONAL. Revista Educación en tecnología. Nos.1,2,3. Bogotá. 1996-1998.

Romero C., Carlos Julio y Ortiz Ch. Evelio Nicanor. Área de tecnología e informática. Universidad Pedagógica Nacional. 1999.

Sagan, Carl. El mundo y sus demonios - La ciencia como una luz en la oscuridad. Santa Fe de Bogotá, Editorial Planeta. 1998. 